\title{
Stability Analysis of Maize Hybrids using Eberhart and Russel Model
}

\author{
H.H. Sowmya ${ }^{1 *}$, M.Y. Kamatar ${ }^{1}$, G. Shanthakumar ${ }^{1}$, S.M. Brunda ${ }^{1}$, \\ T.V. Shadakshari ${ }^{1}$, B.M. Showkath Babu ${ }^{2}$ and Sanjeev Singh Rajput ${ }^{1}$ \\ ${ }^{1}$ Department of Genetics and Plant Breeding, University of Agricultural Sciences, \\ Krishinagar, Dharwad-580005, Karnataka, India \\ ${ }^{2}$ Department of Genetics and Plant Breeding, University of Agricultural Sciences, GKVK, \\ Bengaluru-560065, Karnataka, India \\ *Corresponding author
}

\section{A B S T R A C T}

\section{Keywords}

Maize, Hybrids, Stability, Grain yield, Eberhart and Russel model

Article Info

Accepted:

26 January 2018

Available Online:

10 February 2018
Study was carried out to determine the stability for grain yield of 20 maize hybrids in three environments viz., Dharwad, Sankeshwar and Nippani using Eberhart and Russel model. The single cross hybrids DMH 100-1, DMH 100-2, DMH 100-4, DMH 100-8, DMH 100-12 and DMH 100-16 were promising for majority of characters studied with high mean performance across the locations during summer. The hybrids, DMH 100-1, DMH 100-2, DMH 100-12 and DMH 100-14 were identified as stable over the locations for the monsoon season. The hybrids, DMH 100-1, DMH 100-2 and DMH 100-14 were not influenced much by the season as well as environment and stable across the locations and seasons.

\section{Introduction}

Maize (Zea mays L. 2n=20) is one of the most important crops of world agricultural economy. The crop production is the function of genotype, environment and their interaction (GEI). Significant GEI results in changing behavior of the genotypes across different environments or changes in the relative ranking of the genotypes (Crossa, 1990). A significant $G \times$ E interaction for a quantitative trait such as seed yield can seriously limit the efforts on selecting superior genotypes for improved cultivar development (Kang and
Gorman, 1989). G x E interactions become important when the rank of breeding lines gets changed over environments. This change in rank is called crossover $\mathrm{G}$ x $\mathrm{E}$ interaction (Baker, 1988). Understanding the relationship among yield testing locations is important if plant breeders are to target germplasm better adapted to different production environments or regions (Trethowan et al., 2001). A genotype is considered to be stable if its among-environment variance is small. This is called stability statistic, or a biological concept of stability. A stable genotype possesses an unchanged or least changed 
performance regardless of any variation of the environmental conditions. This concept of stability is useful for quality traits, disease resistance and for stress characters like winter hardiness (Baker and Leon, 1988). In breeding for wide adaptation, the aim is to obtain a variety, which performs well in nearly all environments (Cooper and De-Lacy, 1994). Several stability analyses have been proposed to determine linear relationship between genotypic performance and the environment. Among that Eberhart and Russell (1966) proposed a method in which the environmental index is the mean performance of all entries in an environment. The performance of each genotype is regressed on the environment to obtain its mean performance over all environments. A desirable genotype is one with high mean value, with regression coefficient of 1.0 and deviation from regression is 0 . Such a genotype would have increased performance as the productivity of environment improves. Tollenaar and Lee (2002) reported that highyielding maize hybrids can differ in yield stability and that yield stability and high grain yield are not mutually exclusive. The present study was therefore aimed to evaluate maize hybrids for their stability of performance for yield and yield components across different environments by using Eberhart and Russell (1966) stability model.

\section{Materials and Methods}

To assess the stability performance of 17 single cross maize hybrids (Table 1) for yield and its components experiment was conducted in randomized complete block design (RCBD) with three replications along with 3 checks (Super 900 M, Bio 9681 and Arjun).The experiment was laid out across three different environments viz., Dharwad, Sankeshwar and Nippani and during two different seasons i.e., summer and kharif seasons of 2014. The experiment was laid out, with a row length of $4 \mathrm{~m}$, with inter and intra row spacing of $75 \mathrm{~cm}$ and $20 \mathrm{~cm}$ respectively. Each genotype was sown with 2 rows. Five plants from each replication were randomly selected and tagged for recording observations in each genotype except for days to 50 per cent tasseling and days to 50 percent silking. Data were recorded on different morphological and yield parameters viz., days to anthesis, days to silking, anthesis silking interval, days to brown husking, plant height $(\mathrm{cm})$, cob height $(\mathrm{cm})$, cob length $(\mathrm{cm})$, cob diameter $(\mathrm{cm})$, kernel rows per cob, kernels per row, cob weight/ha $(\mathrm{kg})$, grain yield/ha $(\mathrm{kg})$, test weight (gm) and shelling percentage were recorded in both the seasons at all the experimental sites. The stability of yield performance for each genotype was calculated by regressing the mean yield of individual genotypes on environmental index and calculating the deviations from regressing the mean yield of individual genotypes on environmental index and calculating the deviations from regression as suggested by Eberhart and Russell (1966). Regression coefficient (bi) was considered as an indication of the response of the genotype to varying environment while the environment and genotype $\times$ environment interactions were partitioned into three components viz., environment (linear), genotype $\mathrm{x}$ environment (linear) and deviation from regression (pooled deviation over the genotypes). The stability analysis was done using the linear regression model suggested by Eberhart and Russell (1966).

\section{Results and Discussion}

Pooled analyses of variance, for different quantitative traits over seasons of summer and monsoon as well as across various locations are presented in Table 2 . The results revealed that there was significant difference among the genotypes tested at both???? the 5 and 1 per cent level of significance for all the characters studied except cob height, cob length, kernels per row and shelling percentage in summer season, anthesis to silking interval, plant 
height and kernels per row in monsoon season, while cob height across the locations and seasons. The pooled deviation was also significant for all the characters except days to anthesis, days to silking, anthesis to silking interval, days to brown husking and kernel rows per cob in summer, days to anthesis, days to silking and grain yield in monsoon and anthesis to silking interval across the locations and seasons indicating that non-linear component of $\mathrm{G} \times \mathrm{E}$ interaction was predominant. Three stability parameters viz., mean $(\mathrm{X})$, regression coefficient $\left(\mathrm{b}_{\mathrm{i}}\right)$ and mean square deviation from regression line $\left(\mathrm{S}^{2} \mathrm{di}\right)$ were estimated for all the thirteen traits and the results obtained are presented.

For days to anthesis the mean value over the six environments was 63 days. Among the twenty genotypes twelve showed significant deviation from regression across the locations and seasons, none of the genotypes were stable. However, genotypes DMH 100-12 and DMH 100-15 had less number of days to anthesis with regression value less than one and deviation from regression approaching zero, indicating less sensitivity to environmental changes and were better adapted to poor conditions. For days to silking twelve genotypes (including the checks Arjun and Bio 9681) out of twenty genotypes noticed with significant value for deviation from regression. The hybrid, DMH 100-1 was near to unity for regression coefficient with less number of days to silking and no significant deviation from regression, hence it could be considered as stable over the locations and seasons. The genotypes DMH 100-12 and DMH 100-15 were adoptable to poor environment as it has recorded less number of days to silking, regression value less than one and deviation from regression was approaching zero. For anthesis-silking interval the mean of anthesis to silking interval across the six environments was 2 days and no hybrids recorded with significant value for deviation from regression.

Table.1 List of single cross maize hybrids

\begin{tabular}{|c|c|c|}
\hline Sl. No. & Hybrids & Parents \\
\hline 1 & DMH $100-1$ & DMIL $061 \times$ DMIL 001 \\
\hline 2 & DMH $100-2$ & DMIL $065 \times$ DMIL 096 \\
\hline 3 & DMH $100-3$ & DMIL $069 \times$ DMIL 008 \\
\hline 4 & DMH $100-4$ & DMIL $055 \times$ DMIL 326 \\
\hline 5 & DMH $100-5$ & DMIL $090 \times$ DMIL 051 \\
\hline 6 & DMH $100-6$ & DMIL $326 \times$ DMIL 055 \\
\hline 7 & DMH $100-7$ & DMIL $150 \times$ DMIL 001 \\
\hline 8 & DMH $100-8$ & DMIL $001 \times$ DMIL 318 \\
\hline 9 & DMH $100-9$ & DMIL $136 \times$ DMIL 326 \\
\hline 10 & DMH $100-10$ & DMIL $749 \times$ DMIL 055 \\
\hline 11 & DMH $100-11$ & DMIL $318 \times$ DMIL 150 \\
\hline 12 & DMH $100-12$ & DMIL $055 \times$ DMIL 749 \\
\hline 13 & DMH $100-13$ & DMIL $765 \times$ DMIL 031 \\
\hline 14 & DMH $100-14$ & DMIL $122 \times$ DMIL 112 \\
\hline 15 & DMH $100-15$ & DMIL $147 \times$ DMIL 150 \\
\hline 16 & DMH $100-16$ & DMIL $150 \times$ DMIL 124 \\
\hline 17 & DMH $100-17$ & DMIL $160 \times$ DMIL 103 \\
\hline
\end{tabular}


Table.2 Pooled analysis of variance for stability of fourteen quantitative characters related to yield in Maize across locations and seasons

\begin{tabular}{|c|c|c|c|c|c|c|c|c|c|c|c|c|c|c|}
\hline Source & df & $\begin{array}{l}\text { Days to } \\
\text { anthesis }\end{array}$ & $\begin{array}{l}\text { Days to } \\
\text { silking }\end{array}$ & $\begin{array}{l}\text { Anthesis to } \\
\text { silking } \\
\text { interval }\end{array}$ & $\begin{array}{c}\text { Days to } \\
\text { brown } \\
\text { husking }\end{array}$ & $\begin{array}{c}\text { Plant } \\
\text { height }\end{array}$ & Cob height & $\begin{array}{c}\text { Cob } \\
\text { length }\end{array}$ & $\begin{array}{c}\text { Cob } \\
\text { diameter }\end{array}$ & $\begin{array}{c}\text { Kernel } \\
\text { rows per } \\
\text { cob }\end{array}$ & $\begin{array}{l}\text { Kernels } \\
\text { per row }\end{array}$ & Cob weight & Grain yield & $\begin{array}{c}\text { Shelling } \\
\text { percentage }\end{array}$ \\
\hline Genotype & 19 & $44.25^{* * *}$ & $42.65^{* *}$ & $0.26^{*}$ & $18.82^{* *}$ & $307.65^{* * *}$ & 91.65 & $5.69^{* *}$ & $0.17^{* *}$ & $3.15^{* *}$ & $25.14^{* * *}$ & $7351494^{* *}$ & $4847924^{* * *}$ & $72.47^{*}$ \\
\hline Environment & 5 & $1038.48^{* *}$ & $962.92^{* *}$ & $1.50^{* *}$ & $234.62^{* *}$ & $12152.21^{* *}$ & $4026.52^{* *}$ & $67.06^{* *}$ & $1.00^{* *}$ & $4.50^{* *}$ & $289.55^{* *}$ & $253931677.56^{* *}$ & $130664023.09^{* *}$ & $1048.73^{* *}$ \\
\hline $\begin{array}{c}\text { Variety } \mathbf{x} \\
\text { environment }\end{array}$ & 95 & $4.25^{* *}$ & $4.13^{* *}$ & $0.18^{*}$ & $4.91^{* *}$ & $142.69^{* * *}$ & $55.39^{* *}$ & $2.39^{* *}$ & $0.04^{* *}$ & $0.84^{* *}$ & $11.16^{* *}$ & $2553630^{* *}$ & $1407274^{* *}$ & $42.58^{* *}$ \\
\hline $\begin{array}{c}\text { Environment } \\
\text { (linear) }\end{array}$ & 19 & $5192.42^{* *}$ & $4814.61^{* *}$ & $7.53^{* *}$ & $1173.11^{* *}$ & $60761.04^{* *}$ & $20132.63^{* *}$ & $335.31^{* *}$ & $5.04^{* *}$ & $22.52^{* *}$ & $1447.79^{* *}$ & $1269658387.82^{* *}$ & $653320115.48^{* *}$ & $5243.67^{* *}$ \\
\hline $\begin{array}{c}\text { Variety } \mathbf{x} \\
\text { environment } \\
\text { (linear) }\end{array}$ & 80 & $12.00^{* *}$ & $11.18^{* *}$ & $0.38^{* *}$ & 5.12 & 194.98 & 49.6205 & 3.28 & 0.06 & 0.81 & 13.34 & $4997467^{* *}$ & $2566351^{* *}$ & $67.37^{*}$ \\
\hline Pooled deviation & 228 & $2.20^{* *}$ & $2.25^{* *}$ & 0.13 & $4.61^{* *}$ & $123.14^{* *}$ & $54.00^{* *}$ & $2.06^{* *}$ & $0.04^{* *}$ & $0.80^{* *}$ & $10.08^{* *}$ & $1845537^{* *}$ & $1061629^{* *}$ & $34.57^{* *}$ \\
\hline Pooled error & 119 & 0.62 & 0.60 & 0.14 & 0.51 & 30.16 & 15.65 & 0.45 & 0.01 & 0.35 & 3.21 & 536763.6 & 338087.7 & 10.94 \\
\hline
\end{tabular}

Note: ${ }^{*}=$ significant at $1 \% ; \stackrel{* *}{=}=$ significant at $5 \%$

Table.3a Stability parameters for yield and related traits in maize across three locations and two seasons

\begin{tabular}{|c|c|c|c|c|c|c|c|c|c|c|c|c|c|c|c|c|c|c|c|c|c|}
\hline \multirow[t]{2}{*}{ Genotypes } & \multicolumn{3}{|c|}{ Days to anthesis } & \multicolumn{3}{|c|}{ Days to silking } & \multicolumn{3}{|c|}{$\begin{array}{l}\text { Anthesis to silking } \\
\text { interval }\end{array}$} & \multicolumn{3}{|c|}{$\begin{array}{l}\text { Days to brown } \\
\text { husking }\end{array}$} & \multicolumn{3}{|c|}{ Plant height } & \multicolumn{3}{|c|}{ Cob height (cm) } & \multicolumn{3}{|c|}{ Cob length $(\mathrm{cm})$} \\
\hline & Mean & bi & $\mathbf{S}^{2} \mathrm{di}$ & Mean & bi & $\mathbf{S}^{2} \mathbf{d i}$ & Mean & bi & $\mathbf{S}^{2} \mathbf{d i}$ & Mean & bi & $\mathbf{S}^{2} \mathbf{d i}$ & Mean & bi & $\mathbf{S}^{2} \mathbf{d i}$ & Mean & bi & $\mathbf{S}^{2} \mathbf{d i}$ & Mean & bi & $\mathbf{S}^{2} \mathbf{d i}$ \\
\hline DMH 100 -1 & 60.83 & 1.45 & $2.38^{* *}$ & 63.00 & 1.43 & $3.16^{* *}$ & 2.17 & 1.80 & 0.14 & 88.61 & 1.05 & 0.72 & 172.30 & 1.24 & $44.34^{*}$ & 86.80 & 0.79 & 6.24 & 18.15 & 0.85 & 0.28 \\
\hline DMH 100 -2 & 60.11 & 1.24 & $1.2^{*}$ & 62.28 & 1.26 & $1.96^{* *}$ & 2.17 & 0.59 & -0.03 & 90.17 & 1.16 & $3.34^{* *}$ & 183.90 & 1.00 & -13.34 & 94.76 & 1.02 & -9.34 & 19.98 & 0.03 & $5.23^{* *}$ \\
\hline DMH $100-3$ & 63.56 & 1.10 & $1.1^{*}$ & 65.72 & 1.10 & $1.70^{* *}$ & 2.17 & 1.17 & -0.07 & 88.83 & 1.35 & $7.43^{* *}$ & 188.60 & 1.27 & $69.97^{*}$ & 93.87 & 0.85 & $229.17^{* *}$ & 17.92 & 1.25 & $3.32^{* *}$ \\
\hline DMH $100-4$ & 59.33 & 1.10 & $1.26^{*}$ & 61.56 & 1.07 & 0.62 & 2.22 & 1.98 & 0.02 & 89.44 & 0.90 & $2.73^{* *}$ & 177.40 & 1.26 & $167.57^{* *}$ & 86.99 & 1.16 & $40.89^{* *}$ & 17.61 & 1.27 & $4.13^{* *}$ \\
\hline DMH $100-5$ & 66.72 & 0.95 & 0.49 & 69.06 & 0.92 & 0.16 & 2.33 & 1.71 & 0.18 & 92.83 & 0.72 & $4.29^{* *}$ & 188.40 & 1.25 & $68.69^{*}$ & 95.13 & 1.01 & $40.10^{* *}$ & 16.44 & 1.26 & $1.41^{* *}$ \\
\hline DMH $100-6$ & 66.39 & 1.12 & 0.21 & 68.28 & 1.04 & -0.02 & 1.89 & 3.24 & -0.05 & 91.22 & 0.97 & $4.36^{* *}$ & 190.40 & 0.98 & 25.13 & 90.86 & 0.89 & 12.36 & 17.67 & 1.36 & $2.26^{* *}$ \\
\hline DMH 100 -7 & 65.44 & 0.84 & 0.37 & 67.61 & 0.82 & -0.30 & 2.17 & 1.49 & -0.10 & 93.67 & 0.57 & $1.75^{* *}$ & 193.70 & 0.82 & $113.77^{* *}$ & 96.92 & 1.18 & $67.78^{* * *}$ & 18.63 & 0.68 & 0.68 \\
\hline DMH 100 -8 & 62.94 & 1.45 & $9.05^{* *}$ & 65.17 & 1.43 & $8.23^{* *}$ & 2.22 & 1.84 & -0.10 & 95.06 & 0.90 & 0.49 & 185.60 & 0.99 & $53.29^{*}$ & 97.96 & 0.72 & $23.11^{*}$ & 18.79 & 1.30 & $3.61^{* *}$ \\
\hline DMH $100-9$ & 66.61 & 1.01 & $1.55^{*}$ & 68.83 & 0.98 & $1.75^{* *}$ & 2.22 & 1.74 & -0.07 & 91.33 & 1.27 & $6.25^{* *}$ & 200.10 & 1.04 & $198.64^{* *}$ & 96.72 & 1.32 & $103.72^{* *}$ & 18.93 & 0.87 & 0.43 \\
\hline DMH $100-10$ & 58.72 & 0.89 & $2.9^{* *}$ & 61.39 & 0.94 & $2.50^{* *}$ & 2.67 & -0.32 & 0.06 & 91.06 & 0.65 & $2.29^{* *}$ & 185.40 & $0.416^{* *}$ & -16.50 & 92.52 & 0.97 & -6.49 & 19.41 & 1.28 & $1.06^{*}$ \\
\hline DMH 100 -11 & 59.44 & 0.97 & $3.66^{* *}$ & 62.11 & 1.00 & $3.09^{* *}$ & 2.67 & 0.01 & -0.04 & 90.83 & 1.28 & $1.79^{* *}$ & 186.90 & 0.89 & $47.25^{*}$ & 97.69 & 0.68 & 14.27 & 18.48 & 1.06 & $1.26^{* *}$ \\
\hline DMH $100-12$ & 60.78 & 0.53 & 0.04 & 62.72 & 0.48 & 0.68 & 1.94 & 1.92 & 0.02 & 91.28 & 0.69 & $11.58^{* *}$ & 186.70 & 1.15 & $268.73^{* *}$ & 93.04 & 0.75 & 15.56 & 19.41 & 1.22 & 0.05 \\
\hline DMH 100 -13 & 62.78 & 0.97 & $1.44^{*}$ & 64.72 & 1.00 & $1.46^{* *}$ & 1.94 & 0.56 & 0.06 & 90.56 & 1.32 & -0.45 & 191.90 & 0.52 & $102.63^{* *}$ & 97.52 & 1.06 & $26.880^{*}$ & 18.80 & 0.93 & $1.30^{* *}$ \\
\hline DMH 100 -14 & 64.44 & 1.01 & 0.27 & 66.61 & 1.02 & -0.10 & 2.17 & 0.71 & -0.05 & 91.83 & 0.92 & $16.12^{* *}$ & 196.30 & 0.71 & 36.31 & 99.73 & 0.83 & $23.39^{*}$ & 18.66 & 0.93 & 0.02 \\
\hline DMH 100 -15 & 60.33 & 0.84 & 0.43 & 62.39 & 0.83 & -0.13 & 2.06 & 1.11 & -0.02 & 89.83 & 0.53 & $4.31^{* *}$ & 178.00 & 1.03 & 36.21 & 89.59 & 0.89 & 13.32 & 18.04 & 0.35 & 0.26 \\
\hline DMH 100 -16 & 62.72 & 0.79 & $1.46^{*}$ & 64.56 & 0.80 & $0.94^{*}$ & 1.83 & 0.52 & -0.08 & 90.78 & 1.28 & $4.23^{* *}$ & 193.10 & 0.90 & $69.05^{*}$ & 100.81 & 1.03 & 7.01 & 18.22 & 1.76 & 0.49 \\
\hline DMH 100 -17 & 65.11 & 0.79 & 0.53 & 67.28 & 0.85 & -0.45 & 2.17 & -0.68 & -0.10 & 92.94 & 1.06 & $1.47^{* *}$ & 192.30 & 1.15 & $49.12^{*}$ & 94.99 & 1.28 & 18.71 & 18.36 & 1.22 & 0.38 \\
\hline Arjun & 64.28 & 1.06 & $3.13^{* *}$ & 66.50 & 1.06 & $4.31^{* *}$ & 2.22 & 1.05 & -0.05 & 92.72 & 1.63 & $1.55^{* *}$ & 191.20 & 1.45 & $476.57^{* *}$ & 96.36 & 1.53 & $24.13^{*}$ & 17.34 & 0.02 & 0.44 \\
\hline Super $900 \mathrm{M}$ & 66.61 & 0.98 & 0.17 & 68.83 & 1.05 & 0.43 & 2.22 & -0.86 & -0.07 & 94.17 & 1.02 & $7.36^{* *}$ & 187.8 & 0.96 & $50.23^{*}$ & 96.89 & 0.88 & $48.81^{* *}$ & 18.62 & 1.08 & 0.11 \\
\hline Bio 9681 & 65.22 & 0.90 & $2.32^{* *}$ & 67.44 & 0.91 & $2.93^{* *}$ & 2.22 & 0.43 & -0.08 & 89.56 & 0.76 & 0.32 & 200.8 & 0.98 & 9.67 & 91.10 & 1.17 & $61.78^{* *}$ & 20.94 & 1.26 & $4.97^{* *}$ \\
\hline & 63.12 & & & 65.30 & & & 2.18 & & & 91.34 & & & 188.5 & & & 94.51 & & & 18.52 & & \\
\hline
\end{tabular}


Table.3b Stability parameters for yield and related traits in maize across three locations and two seasons

\begin{tabular}{|c|c|c|c|c|c|c|c|c|c|c|c|c|c|c|c|c|c|c|}
\hline \multirow[t]{2}{*}{ Genotypes } & \multicolumn{3}{|c|}{ Cob diameter $(\mathrm{cm})$} & \multicolumn{3}{|c|}{ Kernel rows per cob } & \multicolumn{3}{|c|}{ Kernels per row } & \multicolumn{3}{|c|}{ Cob weight (kg) } & \multicolumn{3}{|c|}{ Grain yield /ha $(\mathbf{k g})$} & \multicolumn{3}{|c|}{ Shelling percentage } \\
\hline & Mean & bi & $\mathbf{S}^{2} \mathbf{d i}$ & Mean & bi & $\mathbf{S}^{2} \mathbf{d i}$ & Mean & $\mathbf{B i}$ & $\mathbf{S}^{2} \mathbf{d i}$ & Mean & bi & $\mathbf{S}^{2} \mathbf{d i}$ & Mean & bi & $\mathbf{S}^{2} \mathbf{d i}$ & Mean & bi & $\mathbf{S}^{2} \mathrm{di}$ \\
\hline DMH 100 -1 & 4.44 & 2.10 & 0.01 & 15.333 & 1.62 & 0.23 & 33.21 & 0.29 & 4.09 & 6924.43 & $0.78^{*}$ & 471452.04 & 4732.52 & 0.81 & 50095.06 & 69.41 & 1.97 & $62.76^{* *}$ \\
\hline DMH $100-2$ & 4.38 & -0.01 & 0.00 & 14.36 & 0.23 & 0.02 & 37.66 & 0.29 & $7.06^{*}$ & 7579.75 & 0.95 & 461422.95 & 6064.33 & 0.98 & 48620.42 & 80.65 & $-0.002^{*}$ & 9.13 \\
\hline DMH $100-3$ & 4.46 & 1.28 & 0.00 & 15.22 & 1.87 & 0.14 & 33.33 & 1.77 & 3.47 & 7816.57 & 1.05 & 717182.15 & 5604.03 & 1.04 & 34827.61 & 72.84 & 1.09 & -3.64 \\
\hline DMH 100 -4 & 4.27 & 2.04 & $0.02^{*}$ & 14.94 & 1.07 & $0.50^{*}$ & 31.64 & 1.25 & 2.92 & 7271.47 & 1.08 & 125726.44 & 5557.73 & 1.09 & 46965.10 & 77.32 & 0.88 & -9.48 \\
\hline DMH $100-5$ & 4.25 & 1.08 & $0.01^{*}$ & 14.75 & 0.03 & 0.17 & 33.54 & 1.00 & $17.02^{* *}$ & 6153.68 & 1.12 & 494991.21 & 4589.94 & 1.15 & 70276.21 & 74.09 & 0.48 & $22.42^{*}$ \\
\hline DMH 100 -6 & 4.35 & 1.36 & $0.03^{* *}$ & 15.44 & 1.30 & 0.09 & 36.70 & 1.54 & $25.55^{* *}$ & 7943.98 & 1.08 & $081850.86^{*}$ & 6009.95 & 1.20 & $81460.15^{*}$ & 75.42 & 1.28 & 15.29 \\
\hline DMH 100 -7 & 4.49 & 0.45 & $0.01^{*}$ & 16.19 & 2.58 & $1.56^{* *}$ & 33.65 & 0.59 & -1.66 & 5972.16 & 1.01 & 307005.96 & 4251.43 & 0.99 & 39234.21 & 71.97 & 0.98 & 6.16 \\
\hline DMH $100-8$ & 4.64 & 0.50 & $0.05^{* *}$ & 15.84 & 1.99 & 0.14 & 39.09 & 0.63 & -1.83 & 7216.95 & 0.84 & $278553.21^{* *}$ & 4953.02 & 0.83 & $97384.19^{* *}$ & 68.74 & 1.04 & $22.25^{*}$ \\
\hline DMH $100-9$ & 4.32 & 0.92 & $0.01^{*}$ & 14.95 & 0.63 & -0.16 & 37.82 & 1.23 & -1.68 & 8349.57 & 1.04 & $685760.76^{* *}$ & 6039.44 & 1.07 & $24682.16^{* *}$ & 73.08 & $1.51^{*}$ & -6.66 \\
\hline DMH $100-10$ & 4.30 & 0.90 & 0.00 & 14.72 & 1.45 & $0.57^{*}$ & 36.86 & 1.13 & $9.88^{* *}$ & 8292.76 & 1.01 & 529869.78 & 6394.73 & 0.98 & 69533.08 & 78.74 & 0.92 & 4.61 \\
\hline DMH 100 -12 & 4.33 & 0.94 & 0.01 & 15.14 & 0.43 & $0.56^{*}$ & 38.39 & 1.21 & $14.27^{* *}$ & 8048.96 & 0.96 & 151375.16 & 5979.13 & 0.98 & 42563.65 & 75.68 & $1.51^{*}$ & -8.60 \\
\hline DMH $100-13$ & 4.36 & 1.01 & $0.02^{* *}$ & 15.06 & -0.63 & -0.05 & 35.54 & 1.01 & $12.19^{* *}$ & 7828.40 & $1.32^{*}$ & 230884.03 & 6190.36 & $1.38^{* *}$ & 80225.65 & 79.90 & 0.57 & -5.03 \\
\hline DMH 100 -14 & 4.44 & 1.07 & 0.00 & 13.94 & 0.69 & $0.80^{*}$ & 36.19 & 0.73 & 1.80 & 8593.49 & $1.23^{*}$ & 370493.54 & 6417.64 & $1.17^{*}$ & 78093.69 & 76.60 & 1.16 & -5.83 \\
\hline DMH $100-15$ & 4.28 & 1.15 & 0.01 & 15.94 & 0.21 & $0.70^{*}$ & 34.98 & 0.44 & 2.71 & 5241.93 & 0.75 & 230094.03 & 4072.12 & 0.77 & 7845.64 & 78.35 & 0.73 & -0.66 \\
\hline DMH 100 -16 & 4.36 & 1.29 & 0.00 & 15.50 & 1.61 & $0.84^{*}$ & 34.73 & 1.35 & 1.44 & 8856.47 & 1.04 & -22296.78 & 6618.34 & 1.10 & 5072.28 & 75.34 & 1.40 & 2.08 \\
\hline DMH 100 -17 & 4.51 & 1.10 & $0.06^{* *}$ & 14.26 & 2.04 & $0.95^{* *}$ & 33.13 & 1.06 & $17.52^{* *}$ & 7064.10 & 1.46 & 327002.21 ** & 4773.57 & 1.32 & $52735.81^{* *}$ & 69.73 & 0.92 & $44.77^{* *}$ \\
\hline Arjun & 3.86 & 0.56 & $0.27^{* *}$ & 14.27 & -0.25 & 0.08 & 35.19 & 0.47 & 3.02 & 4588.93 & 0.19 & $795069.93^{* *}$ & 3343.11 & 0.09 & $13265.08^{* *}$ & 73.15 & 0.05 & $206.19^{* *}$ \\
\hline Super $900 \mathrm{M}$ & 4.53 & 0.62 & 0.01 & 16.07 & 1.42 & -0.08 & 36.04 & 1.38 & $6.93^{*}$ & 8068.90 & 1.43 & $040558.09^{* *}$ & 6162.06 & 1.35 & $23837.33^{* *}$ & 79.43 & 0.65 & $68.72^{* *}$ \\
\hline Bio 9681 & 4.67 & 0.29 & $0.04^{* *}$ & 16.76 & 1.23 & $1.44^{* *}$ & 36.99 & 1.36 & $6.28^{*}$ & 7429.43 & 0.67 & 671851.64 & 5559.82 & 0.75 & $38876.96^{* *}$ & 75.33 & 1.73 & $24.26^{*}$ \\
\hline & 4.39 & & & 15.21 & & & 35.61 & & & 7338.40 & & & 5441.90 & & & 75.05 & & \\
\hline
\end{tabular}


Across the locations and seasons, genotypes DMH 100-2, DMH 100-3, DMH 100-7, DMH 100-13, DMH 100-14, DMH 100-15 and DMH 100-16 found stable and widely adapted for anthesis to silking interval. On the other hand, three genotypes DMH 100-1, DMH 100-6 and DMH 100-12 were found to be suitable for favorable situations with predictable performance as they passed less anthesis to silking interval along with below average responsiveness $\left(b_{i}>1\right)$ and nonsignificant deviation from regression line. Genotype DMH 100-17 revealed less anthesis to silking interval along with regression coefficient less than unity $\left(b_{i}<1\right)$ and mean deviation from the regression $\left(\mathrm{S}^{2} \mathrm{di}=0\right)$ close to zero indicating that the genotype was specifically adapted to poor environment (Solomon et al., 2008).

On the other hand, one genotype DMH 10013 was found suitable for favorable situations with predictable performance as they possess less anthesis - silking interval along with below average responsiveness $\left(b_{i}>1\right)$ and non-significant deviation from regression line (Kumar et al., 2008). For plant height the highest mean plant height $(200.1 \mathrm{~cm})$ was recorded for DMH 100-9 the tallest and lowest mean plant height $(172.3 \mathrm{~cm})$ was observed for DMH 100-1. The average plant height over the locations and seasons was $188.5 \mathrm{~cm}$. All the hybrids tested were significantly deviating from regression value across the locations and seasons. Over the three locations and two seasons, DMH 100-6 and the best check Bio 9681 was stable across three locations and two seasons for plant height. The average cob height was $94.51 \mathrm{~cm}$ over the three locations and two seasons. Across the locations and seasons, two hybrids viz., DMH 100-2 and the DMH 100-16 performed stable across three locations and two seasons for cob height (Arulselvi, S and Selvi, 2010).
Cob length for DMH 100-2 recorded high mean value $(19.97 \mathrm{~cm})$ and $\mathrm{DMH} 100-5$ noticed with low mean value $(16.44 \mathrm{~cm})$ for cob length, average value over the locations and seasons was $18.52 \mathrm{~cm}$. Across the set of six environments, out of the twenty genotypes tested, DMH 100-7, DMH 100-9, DMH 10012, DMH 100-14, DMH 100-17 and Super $900 \mathrm{M}$ showed higher mean, regression value near to one and non-significant deviation from regression (Karadavut and Akilli, 2012). Hence, these genotypes were stable across the environments for cob length. DMH 100-8 exhibited maximum $(4.64 \mathrm{~cm})$ and DMH $100-$ 5 recorded minimum $(4.25 \mathrm{~cm})$ mean value for cob diameter. Out of the twenty genotypes tested eight genotypes viz., DMH 100-3, DMH 100-10, DMH 100-11, DMH 100-12, DMH 100-13, DMH 100-14, DMH 100-15 and Super $900 \mathrm{M}$ recorded higher mean, regression value near to one and nonsignificant deviation from regression hence, these three genotypes were stable across the environments for days to anthesis. DMH 1001 was adoptable to favorable conditions. The genotypes DMH 100-1, DMH 100-2, DMH 100-6, DMH 100-9, DMH 100-11 and Super $900 \mathrm{M}$ had high mean value with regression coefficient around unity indicating thus adaptability to tested environments for kernel rows per cob (Lenka et al., 2012). The genotypes viz., DMH 100-3 and DMH 100-8 had regression coefficient more than one indicating that these are sensitive to environmental changes and adoptable to favorable environments.

The hybrid DMH 100-8 had high mean number of kernels per row (39.09), while DMH 100-4 had low mean value (31.64). While, over the three locations and two seasons, DMH 100-9, DMH 100-11 and DMH 100-14 recorded higher mean, regression value near to one and nonsignificant deviation from regression (Wasala et al., 2013). Two genotypes DMH 100-8 and 
Arjun exhibited higher mean, regression value less than one and non- significantly deviating from regression, indicating that these genotypes were less sensitive to environmental changes and can be adapted only to poor environments. The mean value for cob weight across three locations and two seasons was $7338.4 \mathrm{~kg} / \mathrm{ha}$. Over six environments, DMH 100-2, DMH 100-3, DMH 100-4, DMH 100-10, DMH 100-11, DMH 100-12, and DMH 100-16 showed higher mean with regression value near to one and non-significant deviation from regression. Hence, these hybrids were stable across the environments for cob weight. The hybrids DMH 100-6, DMH 100-8, DMH 100-9, DMH 100-17, Arjun and Super 900 M had significant deviation from regression indicating that performance of these genotypes is unpredictable. Over six environments, stability analysis for shelling percentage revealed higher mean along with unit regression co-efficient and mean deviation from the regression close to zero in six genotypes viz.,DMH 100-4 and DMH 100-6, DMH 100-10, DMH 100-11, DMH 100-14 and DMH 100-15 indicating that these genotypes are stable across different environments. Genotype DMH 100-16 revealed high mean along with regression coefficient more than unity $\left(b_{i}>1\right)$ and mean deviation from the regression $\left(\mathbf{S}^{2} \mathrm{di}=0\right)$ close to zero indicating that this genotype can be specifically adapted to favorable environments. Further, it was revealed that the genotype DMH 100-13 was specifically adapted to poor environments for shelling percentage as indicated by its high mean, regression co-efficient less than unit $\mathrm{y}$ and deviation from regression close to zero $\left(\mathrm{S}^{2} \mathrm{di}=0\right)$. The average value for grain yield across the locations was $5441.9 \mathrm{~kg} / \mathrm{ha}$. The highest $(6618.3 \mathrm{~kg} / \mathrm{ha})$ and lowest $(4072.12$ $\mathrm{kg} / \mathrm{ha}$ ) mean values were recorded by the genotypes. Across the locations and seasons, seven hybrids DMH 100-2, DMH 100-
3,DMH 100-4, DMH 100-9, DMH 100-10, DMH 100-11, DMH 100-12, DMH 100-14 and $\mathrm{DMH}$ 100-16 were found stable as indicated by their higher mean yield, statistically unit regression and nonsignificant $S^{2}$ di value (Khalil, 2013) The yield performance of the hybrids viz., DMH 100-6, DMH 100-8 and DMH 100-9, DMH 100-13, DMH 100-14, DMH 100-17, Arjun, Super $900 \mathrm{M}$ and Bio 9681 was unpredictable since these hybrids possess significant $S^{2}$ di value. On the basis of stability parameters, the single cross hybrids DMH 100-1, DMH 100-2 and DMH 100-14 were not influenced much by the season/environment were promising for majority of characters studied with high mean performance across the locations and seasons.

The hybrids, these single cross hybrids, which are stable and superior for grain yield could be tested in large scale trials across environments for their wide adaptation in diverse ecological regions. From this, we can stabilize the production level of the crop and it could improve the national production and productivity since, in India production and productivity levels are low by more than 50 percent compared to world productivity.

\section{References}

Arulselvi, S. and Selvi, B., 2010, Grain yield stability of single cross Maize (Zea mays L.) hybrids over three different environments. Electronic J. Pl. Breed., 1(4): 577-584.

Baker, H.C. and J. Leon. 1988. Stability analysis in plant breeding. Plant Breeding, 101: 11-23.

Baker, R.J. 1988. Tests for crossover genotype $\mathrm{x}$ environment interactions. Canadian J. of Plant Sci., 68: 405-410.

Cooper, M. and I.H. De-Lacy. 1994. Relationships among analytical methods used to study genotypic variation and genotype-by-environment interaction in 
plant breeding multi environment experiments. Theoretical and Applied Genetics, 88: 561-572.

Crossa, J. 1990. Statistical analysis of multilocation trials. Adv. in Argon, 44: 55-85.

Dowswell, C. R., Paliwal, R.L. and Cantrell, R.P. 1996. Maize in the third world. Westview Press, Boulder, USA.

Eberhart, S.A. and W.A. Russell. 1966. Stability parameters for comparing varieties. Crop Sci., 6(1): 36- 40.

Kang, M.S. and D.P. Gorman.1989. Genotype $x$ environment interaction in maize. Agron. J., 81(4): 662-664.

Karadavut, U. and Akilli, A., 2012, Genotype-environment interaction and phenotypic stability analysis for yield of corn cultivar. Greener J. Agric. Sci., 2(5): 220-223.

Khalil, 2013, Stability Analysis for Promising Yellow Maize Hybrids under different Locations. Alex. J. Agric. Res. 58: 279- 286.

Kumar, P., Godawat, S. L., Mukesh, Y. and Pandiya, N. K., 2008, Performance of maize (Zea mays) genotypes in different ecological systems. Indian J. Agric.
Sci., 78(11): 939-942.

Lenka, D., Singh, H.C., Gupta, G.R. and Singh, A. K., 2012, Screening of stable single cross hybrids in maize (Zea mays L.). Adv. Life Sci., 1(1): 72-74.

Shaw, R. H., 1988, Climate requirement. In: (Eds. G. F. Sprague and J. W. Dudley) Corn and corn improvement. Amer. Soc. Agron. Madison, WI. pp. 609-633.

Solomon, A., Nigussie, M. and Zelleke, H., 2008, Genotype - environment interaction and stability analysis for grain yield of maize (Zea mays L.) in Ethiopia. Asian J. Plant Sci., 7(2): 163169.

Trethowan, R.M., J. Crossa, M. Ginkel and S. Rajaram. 2001. Relationships among Bread Wheat International Yield Testing Locations in Dry Areas. Crop Sci., 41: 1461-1469.

Wasala, S. K., Guleria, S.K., Sekhar, J.C., Mahajan, V., Srinivasan, K., Prasad, R. and Prasanna, B. M., 2013, Analysis of yield performance and genotype $\times$ environment effects on selected maize (Zea mays L.) landrace accessions of India. Indian J. Agric. Sci., 83 (3): 287293.

\section{How to cite this article:}

Sowmya, H.H., M.Y. Kamatar, G. Shanthakumar, S.M. Brunda, T.V. Shadakshari, B.M. Showkath Babu and Sanjeev Singh Rajput. 2018. Stability Analysis of Maize Hybrids using Eberhart and Russel Model. Int.J.Curr.Microbiol.App.Sci. 7(02): 3336-3343. doi: https://doi.org/10.20546/ijcmas.2018.702.399 